ARTICLE

https://doi.org/10.1038/s41467-019-09154-5

\title{
Intramolecular electron-induced proton transfer and its correlation with excited-state intramolecular proton transfer
}

Wei Wang ${ }^{1}$, Mary Marshall (id ${ }^{2}$, Evan Collins ${ }^{2}$, Sara Marquez², Chaonan Mu¹, Kit H. Bowen² \& Xinxing Zhang ${ }^{1}$

Electron-induced proton transfer depicts the proton motion coupled with the attachment of a low-energy electron to a molecule, which helps to understand copious fundamental chemical processes. Intramolecular electron-induced proton transfer is a similar process that occurs within a single molecule. To date, there is only one known intramolecular example, to the best of our knowledge. By studying the 10-hydroxybenzo[h]quinoline and 8-hydroxyquinoline molecules using anion photoelectron spectroscopy and density functional theory, and by theoretical screening of six other molecules, here we show the intramolecular electroninduced proton transfer capability of a long list of molecules that meanwhile have the excitedstate intramolecular proton transfer property. Careful examination of the intrinsic electronic signatures of these molecules reveals that these two distinct processes should occur to the same category of molecules. Intramolecular electron-induced proton transfer could have potential applications such as molecular devices that are responsive to electrons or current.

\footnotetext{
${ }^{1}$ Key Laboratory of Advanced Energy Materials Chemistry (Ministry of Education), College of Chemistry, Nankai University, 300071 Tianjin, China.

2 Department of Chemistry, Johns Hopkins University, Baltimore, MD 21218, USA. Correspondence and requests for materials should be addressed to K.H.B. (email: kbowen@jhu.edu) or to X.Z. (email: zhangxx@nankai.edu.cn)
} 
E lectron-induced proton transfer (EIPT) describes the proton motion coupled with the attachment of a low-energy electron to a molecule. It widely occurs in nature and has been observed in many different systems ${ }^{1-20}$. EIPT helps to understand copious fundamental chemical processes such as acid-base chemistry: it is known that in some simple acid-base reactions, the proton transfer from one acid molecule (e.g. $\mathrm{HCl}, \mathrm{HBr}, \mathrm{HI}$ and $\mathrm{H}_{2} \mathrm{O}$ ) to another base molecule (e.g. $\mathrm{NH}_{3}$ and pyridine) cannot occur. The attachment of an electron otherwise facilitates these reactions due to the formation of a transient dipole bound anionic state $^{1,2}$ or a unstable valence bound anionic state ${ }^{3}$. EIPT is also considered to be accountable for the damage of DNA in cancer radiotherapy ${ }^{4,5}$. Secondary electrons generated by high-energy photons attach to DNA molecules and induce EIPT between the double strands. As a result, normal and cancerous cells are killed indistinguishably. In order to understand DNA damage by lowenergy electrons at the molecular level, gas phase isolated model systems using DNA subunits such as nucleobases and other biologically relevant molecules have been studied in anion beams spectrometrically and spectroscopically. These model systems include EIPT between guanine and cytosine ${ }^{6}$, uracil and alanine ${ }^{7}$, uracil and glycine ${ }^{8}$, thymine and glycine $e^{9}$, uracil dimer ${ }^{10}$, thymine dimer ${ }^{10}$, adenine and 9-methyladenine ${ }^{11}$, adenine and thymine ${ }^{12}$, 9-methyladenine and 1-methylthymine ${ }^{13}$, formic acid and adenine ${ }^{13}$, formic acid and 1-methylcytosine ${ }^{14}$, formic acid and thymine ${ }^{15}$, formic acid and uracil ${ }^{15}$, uracil and alcohols ${ }^{16}$, uracil and $\mathrm{H}_{2} \mathrm{~S} / \mathrm{H}_{2} \mathrm{Se} / \mathrm{HCN}^{17,18}$. Conclusively, EIPT is vital in understanding many fundamental chemical and biological processes in nature.

All of the above-mentioned EIPT examples, nevertheless, occur between at least two molecules, i.e. they are intermolecular EIPT. Here we show some other molecules with their local structures satisfying certain conditions could undergo intramolecular EIPT (iEIPT). Reactions that occur within one molecule, which is the smallest environment that a chemical reaction could have, are interesting for its own sake. It can be inferred that a certain molecule should satisfy at least three criteria to undergo iEIPT: (1) it should have one functional group as the proton donor (such as $-\mathrm{OH}$ or $-\mathrm{COOH}$ ) and one functional group as the proton receptor (nucleophiles such as lone pair); (2) these two functional groups should be in close proximity and exhibit a pre-existing hydrogen bond; (3) this molecule should be able to electronically accommodate one excess electron in a manner of a $\pi^{*}$ antibonding orbital. Historically, iEIPT was only observed in the acetoacetic acid anion ${ }^{20}$ whose neutral counterpart fulfills the above three criteria. To systematically search for molecules of this kind, we examined another class of molecules having the ability of excited-state intramolecular proton transfer (ESIPT) ${ }^{21-42}$. Numerous experimental and theoretical efforts have been paid to ESIPT owing to its applications ${ }^{33-38}$ in molecular probes, luminescent materials, molecular logic gates, etc. It is not surprising that all of the ESIPT molecules fulfill the above three criteria of iEIPT. With the help from the well-documented ESIPT molecules, much more iEIPT molecules could be potentially discovered. Nevertheless, ESIPT involves the excited states of neutral molecules while EIPT deals with anionic systems; they are very different in nature at the first glance. Therefore, even if these two categories of proton transfer happen to occur in the same molecules, the intrinsic property that facilitates this "coincidence" is yet to be discovered.

Here, we report the iEIPT capability of 10-hydroxybenzo[h] quinoline (HBQ) and 8-hydroxyquinoline (HQ), both of which have been shown to display ESIPT properties ${ }^{24-32}$, especially the former, HBQ, was extensively studied both experimentally and theoretically ${ }^{24-31}$. Here the iEIPT of these two molecules are studied by mass spectrometry and photoelectron spectroscopy, and the experimental results are then validated by density functional theory (DFT) calculations. We also performed a theoretical screening of six other ESIPT molecules, all of which could potentially undergo iEIPT upon electron attachment. More importantly, why ESIPT and iEIPT should occur to the same category of molecules are discussed by studying their local electronic properties. With the examples and evidences provided in the current study, we shed light on a huge list of iEIPT molecules, which promise to have applications such as molecular electronic devices that are responsive to electrons or current.

\section{Results}

Photoelectron spectroscopy. Figure 1 presents the photoelectron spectra of $\mathrm{HBQ}^{-}$and $\mathrm{HQ}^{-}$taken with $532 \mathrm{~nm}$ laser $(2.33 \mathrm{eV})$. Both of the spectra possess two electron binding energy (EBE) bands, they corresponding to the non-proton-transferred (Non$\mathrm{PT}$ ) and the proton-transferred (PT) isomers. All of the EBE bands in these two spectra exhibit vibrational progressions of $\sim 0.15 \mathrm{eV}$, which are attributable to the transitions from the anion's ground state to different vibrational states of the neutral isomers (vide infra). For the $\mathrm{HBQ}^{-}$spectrum, the Non-PT band starts from $0.45 \mathrm{eV}$ and peaks at $0.68 \mathrm{eV}$, and the PT band starts from around $0.85 \mathrm{eV}$ (value obtained by extrapolating the left EBE side) and peaks at $1.26 \mathrm{eV}$. Therefore, the experimental vertical detachment energies (VDE) of the Non-PT and PT $\mathrm{HBQ}^{-}$isomers, i.e. the peak values of each EBE band, are 0.68 and $1.26 \mathrm{eV}$, respectively. For the $\mathrm{HQ}^{-}$spectrum, the Non-PT band starts from $0.2 \mathrm{eV}$ and peaks at $0.48 \mathrm{eV}$, and the PT band starts from $0.95 \mathrm{eV}$ (by extrapolation) and peaks at $1.12 \mathrm{eV}$. Hence, the experimental VDE values of the Non-PT and PT HQisomers are 0.48 and $1.12 \mathrm{eV}$, respectively. All of these VDE values are tabulated in Table 1 for comparison with the calculated results. Note that the $\mathrm{PT}$ band of $\mathrm{HBQ}^{-}$is more intense than the Non-PT band, and $\mathrm{HQ}^{-}$shows the opposite. The PT band is also

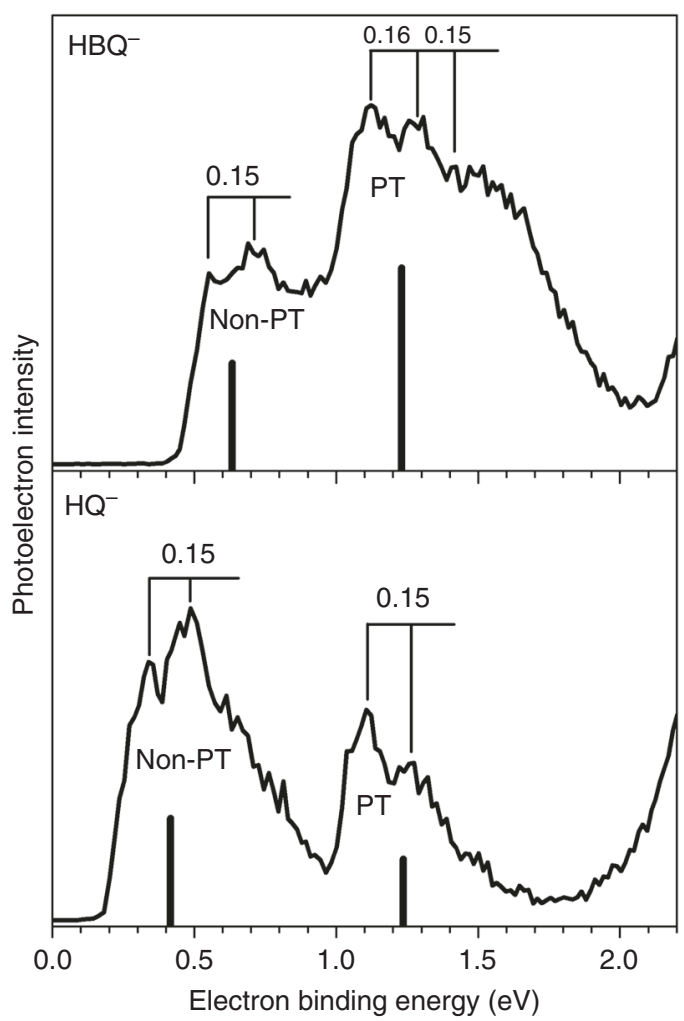

Fig. 1 Photoelectron spectroscopy. Photoelectron spectra of $\mathrm{HBQ}^{-}$ and $\mathrm{HQ}^{-}$taken with a $532 \mathrm{~nm}$ laser. $\mathrm{PT}$ proton-transferred, Non-PT non-proton-transferred 


\begin{tabular}{|c|c|c|c|c|c|c|}
\hline & & Experimental VDE & Theoretical VDE & Expt. ADE & Theo. ADE & Theo. EA \\
\hline$\overline{\mathrm{HBQ}^{-1 / 0}}$ & Non-PT $\mathrm{HBQ}^{-}$ & 0.68 & 0.634 & 0.45 & 0.377 & 0.463 \\
\hline
\end{tabular}

broader than the Non-PT band for $\mathrm{HBQ}^{-}$but those of $\mathrm{HQ}^{-}$look similar; these observations will be explained in the following discussions. The electron affinity (EA) of a molecule is defined as the energy difference between the ground state of the anion and the ground state of the neutral. Only if when there is enough Franck-Condon overlap between these two states can EA be experimentally observed. In regard of this, the EAs of both HBQ and HQ cannot be observed in the spectra, and this statement will be discussed with the calculated potential energy surfaces in the following paragraphs. The adiabatic detachment energy (ADE) of an anion is defined as the energy difference between a certain anionic isomer and the neutral relaxed to the anion's nearest local minima. In the current specific case, the ADE of the Non-PT anion is the energy difference between the Non-PT anion and the Non-PT neutral, and the ADE of the PT anion is the energy difference between the PT anion and the PT neutral, if there is a PT neutral at all.

Theoretical results. Figure 2 shows the structures of the Non-PT and $\mathrm{PT} \mathrm{HBQ}^{-}$and $\mathrm{HQ}^{-}$anions with their corresponding highest occupied molecular orbitals (HOMO, where the excess electron dwells) displayed aside. The relative energies corrected by the zero-point energies (ZPE) are also presented. The 3D coordinates of all the species calculated are provided in Supplementary Table 1. For both $\mathrm{HBQ}^{-}$and $\mathrm{HQ}^{-}$, the $\mathrm{PT}$ isomers have lower energy than the Non-PT ones, suggesting that proton transfer is thermodynamically favored. The singly occupied HOMOs of all these four systems are delocalized $\pi^{*}$ antibonding orbitals, which satisfy the third criterion of searching for iEIPT molecules and also explain the stability of the anions. Both HBQ and HQ undoubtedly fulfill the first and second criteria based on their geometric nature.

Figure 3 exhibits the potential energy surfaces of neutral and anionic HBQ (Fig. 3a) and HQ (Fig. 3b) along the $\mathrm{O}-\mathrm{H}$ bond using the relaxed scan method. For each scan step, only the $\mathrm{O}-\mathrm{H}$ bond distance is fixed and the rest of the molecule relaxes. The neutral potential energy surface is given by a dashed line and the anion by a solid line. For HBQ, the neutral only has one potential energy well, corresponding to the Non-PT neutral HBQ. The PT neutral does not exist. This observation confirms that the proton transfer in the anion was indeed induced by the excess electron since the neutral does not naturally have the PT isomer, or alternatively stated, EIPT did occur. For the anion potential energy surface, two wells were discovered, corresponding to the Non-PT and PT isomers. The low barrier between the two wells, $0.06 \mathrm{eV}$, together with the fact that PT isomer is thermodynamically favored compared to the Non-PT isomer, justify the observation of the more intense PT band. Here we conclude the mechanism of forming the $\mathrm{PT} \mathrm{HBQ}^{-}$as follows: Non-PT neutral HBQ obtains an electron, giving the Non-PT $\mathrm{HBQ}^{-}$, which partially undergoes iEIPT to yield the $\mathrm{PT}_{\mathrm{HBQ}}{ }^{-}$. The anionic and neutral HBQ structures corresponding to each well are also embedded in Fig. 3a. The transition state (TS) between the Non-PT and PT isomers displays a six-member ring with the proton in the middle of the oxygen and nitrogen atoms. For the neutral molecule, we also present the calculated vibrational mode with the displacement vectors; this motion is the $\mathrm{C}-\mathrm{H}$ and $\mathrm{O}-\mathrm{H}$ shear mode, calculated to be $1319 \mathrm{~cm}^{-1}$, close to the experimental value, $0.15 \mathrm{eV}$. The experimental ADE of the Non-PT anion is the energy difference between the Non-PT anion and Non-PT neutral, corresponding to the onset of the Non-PT band in the photoelectron spectrum, $0.45 \mathrm{eV}$, in good consistency with the single point calculation value with $\mathrm{ZPE}$ correction $(0.377 \mathrm{eV})$. The $\mathrm{ADE}$ of $\mathrm{PT} \mathrm{HBQ}^{-}$, however, cannot be observed by experiment, because there is not a neutral potential well above the $\mathrm{PT} \mathrm{HBQ}^{-}$, and the nearest neutral minimum is still the NonPT neutral. So the ADE of the $\mathrm{PT} \mathrm{HBQ}^{-}$should be the energy difference between the Non-PT neutral and the PT anion. Due to the lack of Franck-Condon overlap, the extrapolated onset of the PT band $(0.85 \mathrm{eV})$ in the spectrum is much higher than the calculated ADE $(0.463 \mathrm{eV})$. The lack of Franck-Condon overlap also explains why the PT band is much wider than the Non-PT band in the spectrum. According to Fig. 3a, the EA of HBQ should be the energy difference between the PT anion and the Non-PT neutral, this value being the same as the ADE of the PT anion, which cannot be observed by experiment, neither, so we only report the theoretical EA result, $0.463 \mathrm{eV}$, in Table 1 .

Figure $3 \mathrm{~b}$ shows the potential energy surfaces of neutral and anionic $\mathrm{HQ}$ along the $\mathrm{O}-\mathrm{H}$ bond, where both the anion and the neutral have two wells, corresponding to Non-PT and PT isomers, respectively. The existence of the PT isomer of the neutral gives rise to the experimental observation of the ADE of the $\mathrm{PT} \mathrm{HQ}^{-}$(extrapolated to be $0.95 \mathrm{eV}$ ), in good agreement with the calculated result $(0.997 \mathrm{eV})$. The experimental ADE of the Non-PT $\mathrm{HQ}^{-}, 0.2 \mathrm{eV}$, also agrees well with the calculated 0.177 $\mathrm{eV}$. The photodetachment from $\mathrm{PT} \mathrm{HQ}^{-}$to $\mathrm{PT} \mathrm{HQ}$ has a very good Franck-Condon overlap, resulting in a narrower PT band in the photoelectron spectrum compared to the $\mathrm{PT} \mathrm{HBQ}^{-}$case. The barrier between Non-PT and $\mathrm{PT} \mathrm{HQ}^{-}, 0.45 \mathrm{eV}$, is much higher than that of $\mathrm{HBQ}^{-}(0.06 \mathrm{eV})$; this is because the fivemember ring $\mathrm{TS}$ of $\mathrm{HQ}^{-}$has more tension than a six-member ring. This higher barrier also explains the relative lower intensity of $\mathrm{PT}_{\mathrm{HQ}}{ }^{-}$band compared to the Non-PT $\mathrm{HQ}^{-}$band in the spectrum. Due to this relatively higher barrier, the PT isomer should increase with increased reaction time. With a longer delay between the photoemission laser in the ion source and the ion extraction voltage, the Non-PT isomer is given a longer time for the proton to transfer. Accordingly, the PT isomer's intensity increases with the longer delay (Supplementary Figure 1). A similar observation was made in our previous studies ${ }^{39}$. The EA of HQ should be the energy difference between PT $\mathrm{HQ}^{-}$and Non-PT HQ, again, due to insufficient Franck-Condon overlap, we only report the calculated EA value, $0.253 \mathrm{eV}$, in Table 1. For the neutral $\mathrm{HQ}$, the $\mathrm{PT}$ isomer is $0.74 \mathrm{eV}$ higher in energy compared to the Non-PT isomer, which makes HQ mostly NonPT at room temperature. Hence, the mechanism of iEIPT of HQ is similar to that of HBQ: Non-PT HQ obtains an electron, giving 


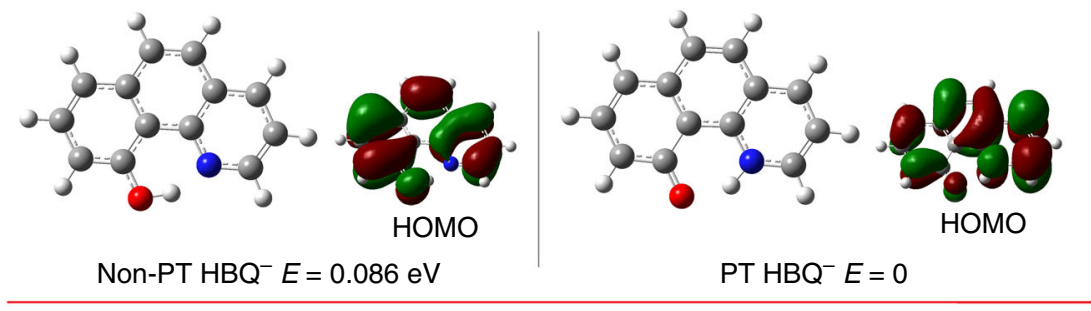

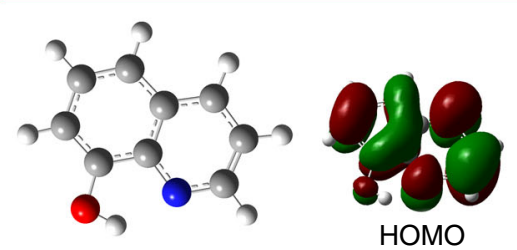

Non-PT $\mathrm{HQ}^{-} E=0.076 \mathrm{eV}$

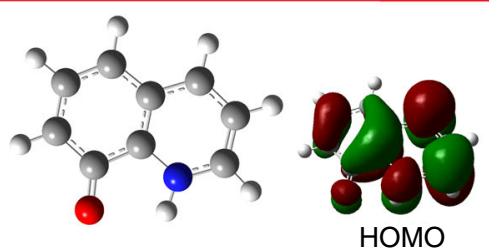

$\mathrm{PT} \mathrm{HQ}^{-} E=0$

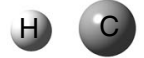

Fig. 2 Calculated structures. Calculated structures of $\mathrm{PT}$ and Non-PT $\mathrm{HBQ}^{-}$and $\mathrm{HQ}^{-}$, with their relative energies and $\mathrm{HOMOs}$ listed alongside. PT protontransferred; Non-PT non-proton-transferred
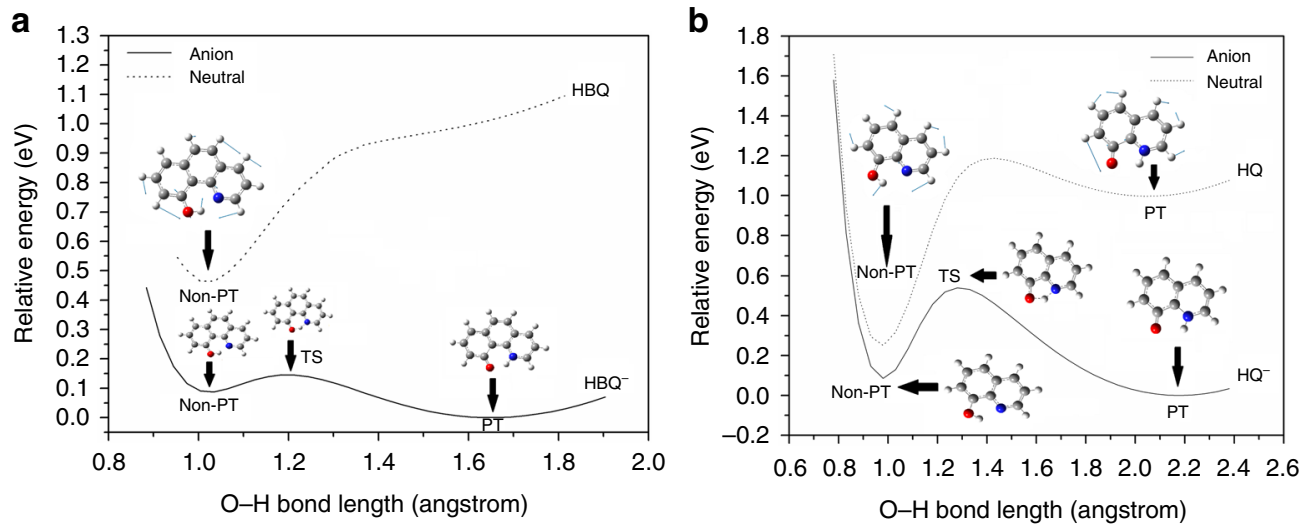

Fig. 3 Potential energy surfaces. Potential energy surfaces of $\mathrm{HBQ}^{\mathrm{O} /-}(\mathbf{a})$ and $\mathrm{HQ}^{\mathrm{O} /-}(\mathbf{b})$ along the $\mathrm{O}-\mathrm{H}$ coordinate using the relaxed scan method. $\mathrm{PT}$ proton-transferred, Non-PT non-proton-transferred, TS transition state

the Non-PT HQ ${ }^{-}$, which further undergoes iEIPT to yield the PT $\mathrm{HQ}^{-}$. Again, we present the displacement vectors of the experimentally observed vibrational progressions for both NonPT and PT neutrals. Calculated frequencies of the Non-PT $\left(1265 \mathrm{~cm}^{-1}\right)$ and PT $\left(1191 \mathrm{~cm}^{-1}\right)$ neutrals agree well with the experimental values $(\sim 0.15 \mathrm{eV})$.

In biomedia, an environment full of water, the potential energy surfaces of the anionic species in Fig. 3 will be significantly lowered compared to their neutral counterparts due to stronger hydrogen bonding with the negative charge. Therefore, solvation stabilizes these anionic species, which often results in blue shifts in the photoelectron spectra (see e.g. ref. ${ }^{3}$ ), making these anionic species more likely to be present in vivo.

To sum, due to the coexistence of the Non-PT and PT anionic isomers and the fact that neutral molecules naturally exist in the Non-PT form, it can be concluded that the stepwise mechanism of iEIPT is: Non-PT neutral $\rightarrow$ Non-PT anion $\rightarrow$ PT anion.

\section{Discussion}

Experimental and theoretical evidences have been provided in the current study to reveal the iEIPT capability of HBQ and HQ. This work also aims to provide evidences for the correlation between iEIPT and ESIPT. Here the iEIPT potential of six other molecules is screened at the $\omega \mathrm{B} 97 \mathrm{XD} / 6-31+\mathrm{G}(\mathrm{d}, \mathrm{p})$ level of theory, they being 2-(2'-pyrydyl) phenol, o-hydroxyphenyl-(1,3)diazine, o-hydroxyphenyl-(1,3,5)triazine, 2 -(2'-hydroxyphenyl) benzimidazole, 1-hydroxy-9H-fluoren-9-one and 3-hydroxyflavone, all of which are also ESIPT molecules $24,36,40-42$. Figure 4 presents the PT and Non-PT anionic isomers and their relative energies of these six molecules. For 2-(2'-pyrydyl) phenol and o-hydroxyphenyl- $(1,3)$ diazine, the PT isomers have lower energy than the Non-PT ones, indicating that EIPT is favored energetically. For o-hydroxyphenyl- $(1,3,5)$ triazine, 2 - $\left(2^{\prime}\right.$-hydroxyphenyl) benzimidazole, 1-hydroxy-9H-fluoren-9-one and 3-hydroxyflavone, the PT isomers have slightly higher energy than the Non-PT ones. Evidenced by HQ and HBQ, the relative higher or lower energy of $\mathrm{PT}$ and Non-PT isomers does not necessarily eliminate a certain isomer's existence, and they should by and large coexist upon receiving the excess electron.

Next we discuss the obvious question: apart from the three basic criteria, why do ESIPT and EIPT occur to the same group of molecules? In Fig. 5, we brief the mechanism of ESIPT along the purple arrows, the mechanism of iEIPT along the brown arrows, and the molecule of interest is simplified as an anomalous circle to denote the body of a $\pi$-conjugate system, with a $\mathrm{Nu}$ : to denote the proton receptor and an $-\mathrm{OH}$ group to denote the proton donor. Robb and his coworkers ${ }^{40}$, as well as Sobolewski and Domcke ${ }^{43}$ separately performed detailed computational studies of the ESIPT mechanism. They all pointed out that the transient 


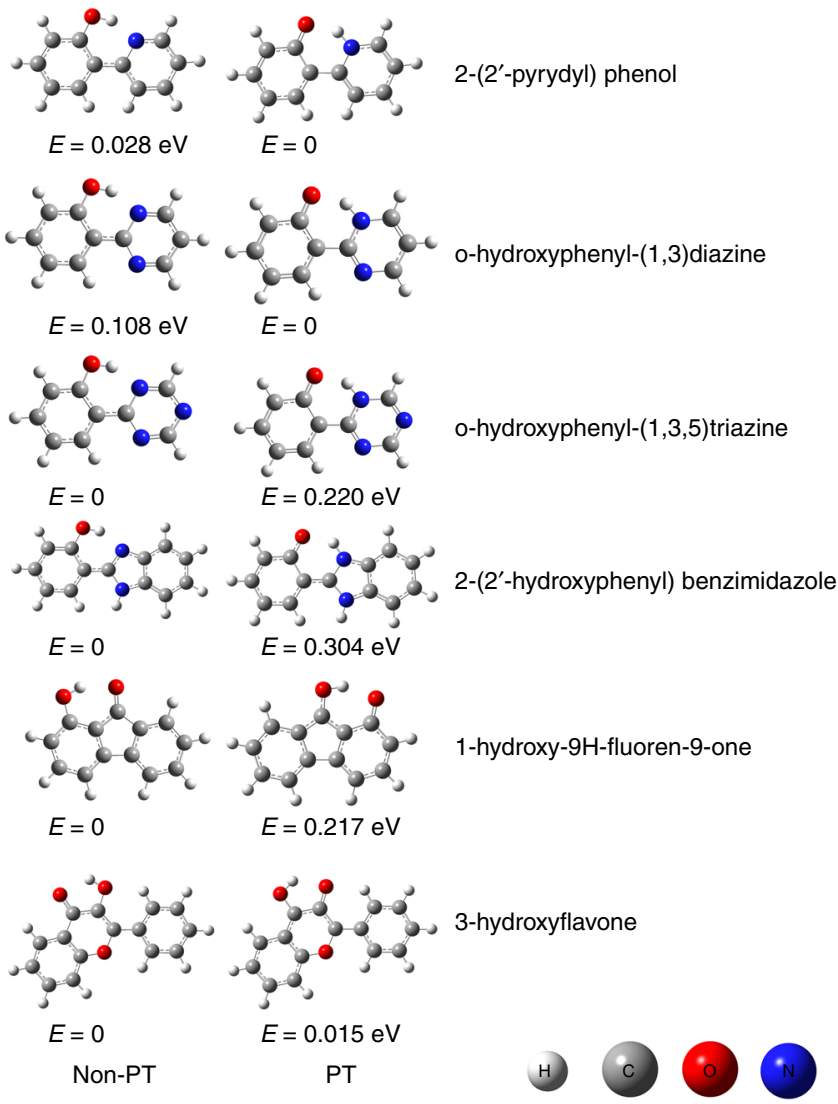

Fig. 4 Molecular anion structures and relative energies. Structures and relative energies of the PT and Non-PT forms of several molecular anions. PT proton-transferred, Non-PT non-proton-transferred

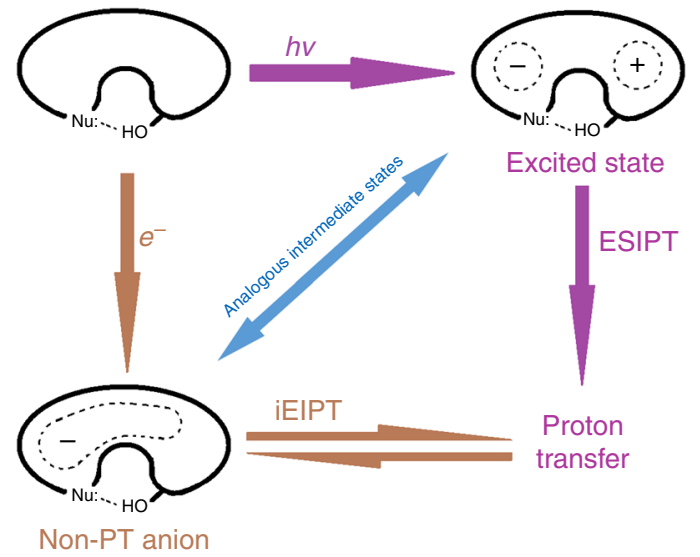

Fig. 5 Proposed mechanism. Mechanisms and correlation of excited-state intramolecular proton transfer (ESIPT) and intramolecular electron-induced proton transfer (iEIPT). Nu nucleophile, Non-PT non-proton-transferred

excited state of the neutral, key to the proton transfer, is a chargetransfer state, where the proton receptor part of the molecule is negatively charged and the donor part is positively charged. Hence, the proton's automatic transfer to the negatively charged site of the molecule is driven by the charge separation. In the iEIPT case along the brown arrows, upon electron attachment, the negative charge is delocalized on the ring of the Non-PT isomer; however, it is more localized on the more electronegative $\mathrm{Nu}$ : side. In the current case, Nu: is the nitrogen atom, and it draws $-0.63 e$ in Non-PT HBQ ${ }^{-}$and $-0.67 e$ in Non-PT HQ ${ }^{-}$as revealed by the natural population analysis (NPA). The NPA charges of the proton receptor sites of those species in Fig. 4 are tabulated in Supplementary Table 2, all of which exhibit high negative charge (from -0.5 to $-0.8 e$ ). The Non-PT anionic state can be viewed as an intermediate state towards PT, which has the unevenly distributed negative charge and "stimulates" the transfer of the proton to the more negatively charged side of the ring, i.e. the Nu:. Apparently, the Non-PT anionic state in EIPT is analogous to the excited state in ESIPT, explaining why these two rather distinct processes should occur to the same group of molecules. Remarkably, Sobolewski and Domcke also opined that in the intermolecular excited-state proton transfer processes, the charge transfer of the transient excited state was again the driving force of proton transfer ${ }^{43}$, which is also analogous to the intermolecular EIPT examples we previously studied ${ }^{1-3,6-19}$.

To conclude, in this work we provide evidence for the correlation between ESIPT and iEIPT molecules: if a molecule could undergo ESIPT, it could well be able to undergo iEIPT. Experimental and theoretical evidences are given to support the iEIPT properties of two ESIPT molecules, HBQ and HQ. Theoretical calculations investigate the viability of iEIPT for six other ESIPT molecules. This remarkable correlation between iEIPT and ESIPT processes arises from their similar states right before the proton transfer step, where the proton receptor side of the molecule is more negatively charged; as a result, the proton transfers. The discovery of the long list of iEIPT molecules could potentially have the applications as molecular devices (e.g. molecular switch) that are sensitive to stimuli such as electrons or current.

\section{Methods}

Experimental methods. Anion photoelectron spectroscopy is conducted by crossing a mass-selected beam of negative ions with a fixed-frequency photon beam and energy-analyzing the resultant photodetached electrons. It is governed by the energy-conserving relationship, $h v=\mathrm{EBE}+\mathrm{EKE}$, where $h v$ is the photon energy, $\mathrm{EBE}$ is the electron binding (transition) energy, and EKE is the electron kinetic energy. Our anion photoelectron spectrometer, which has been described previously ${ }^{44}$, consists of a laser vaporization anion source, a linear time-of-flight mass analyzer/selector, a pulsed Nd:YAG photodetachment laser, and a magnetic bottle electron energy analyzer. Photoelectron spectra were calibrated against the wellknown photoelectron spectrum of $\mathrm{Cu}^{-}{ }^{45}$. Both HBQ and HQ were purchased from Alfa Aesar ${ }^{\circledast}$ and used without further purification. Parent anions of HBQ and HQ were generated in a photoemission ion source. Briefly, a copper rod was interrogated by a pulsed Nd:YAG laser beam operating at a wavelength of $532 \mathrm{~nm}$ to photoemit electrons. HBQ or HQ was slightly heated to $40^{\circ} \mathrm{C}$ in an oven placed in between the photoemission hosing and a pulsed valve, which supersonically expands a plume of ultrahigh purity helium gas (backing pressure $100 \mathrm{psi}$ ) to cool and carry the plasma. Negatively charged anions were then extracted into the spectrometer prior to mass selection and photodetachment. The delay between the photoemission laser in the ion source and the ion extraction voltage is varied to allow different proton transfer time periods in order to distinguish between isomers.

Theoretical methods. DFT calculations were conducted by applying the hybrid meta-GGA functional $\omega$ B97XD using the Gaussian09 software package to determine the geometries of both neutral and anionic isomers $\mathrm{HBQ}$ and $\mathrm{HQ}$, the adiabatic electron affinities (EA), the ADE, the VDE and the potential energy surface along the $\mathrm{O}-\mathrm{H}$ bond. All geometries, including that of the anion and its corresponding neutral molecule, were fully optimized without any geometrical constraints using the $6-31++\mathrm{G}(\mathrm{d}, \mathrm{p})$ basis set and then improved by single point energy calculations with a larger basis set $6-311++\mathrm{G}$ (3df, 3pd). EA is calculated for the neutral molecules. ADE and VDE were calculated for both the protontransferred and non-proton-transferred anionic isomers. The potential energy surface was scanned along the $\mathrm{O}-\mathrm{H}$ coordinate with a step width of $0.05 \AA$ by sequentially relaxing the rest of the molecule to its ground state to reveal the barrier height and potential wells along the proton transfer pathway. Natural population analysis was utilized to reveal the atomic charges in the species of interest.

\section{Data availability}

The authors declare that the data supporting the findings of this study are available within the paper and its supplementary information files, and from the authors upon reasonable request. 
Received: 8 November 2018 Accepted: 20 February 2019

Published online: 12 March 2019

\section{References}

1. Eustis, S. N. et al. Electron-driven acid-base chemistry: proton transfer from hydrogen chloride to ammonia. Science 319, 936 (2008).

2. Eustis, S. N., Whiteside, A., Wang, D., Gutowski, M. \& Bowen, K. H. Ammonia-hydrogen bromide and ammonia-hydrogen iodide complexes: anion photoelectron and ab initio studies. J. Phys. Chem. A 114, 1357-1363 (2010).

3. Wang, Y. et al. The onset of electron-induced proton-transfer in hydrated azabenzene cluster anions. Phys. Chem. Chem. Phys. 18, 704-712 (2016).

4. Sanche, L. Low energy electron-driven damage in biomolecules. Eur. Phys. J. D. 35, 367-390 (2005)

5. Kumar, A. \& Sevilla, M. D. Proton-coupled electron transfer in DNA on formation of radiation-produced ion radicals. Chem. Rev. 110, 7002-7023 (2010).

6. Szyperska, A. et al. Low-energy-barrier proton transfer induced by electron attachment to the guanine $\cdots$ cytosine base pair. Chemphyschem 11, 880-888 (2010).

7. Dąbkowska, I. et al. Barrier-free intermolecular proton transfer induced by excess electron attachment to the complex of alanine with uracil. J. Chem. Phys. 120, 6064-6071 (2004)

8. Gutowski, M. et al. Barrier-free intermolecular proton transfer in the uracilglycine complex induced by excess electron attachment. Eur. Phys. J. D. 20 431-439 (2002).

9. Dabkowska, I. et al. Barrier-free proton transfer in anionic complex of thymine with glycine. Phys. Chem. Chem. Phys. 6, 4351-4357 (2004).

10. Ko, Y. J. et al. Photoelectron spectroscopy of homogeneous nucleic acid base dimer anions. Phys. Chem. Chem. Phys. 12, 3535-3541 (2010).

11. Mazurkiewicz, K. et al. Valence anions in complexes of adenine and 9ethyladenine with formic acid: stabilization by intermolecular proton transfer. J. Am. Chem. Soc. 129, 1216-1224 (2007).

12. Radisic, D. et al. AT base pair anions versus (9-Methyl-A)(1-Methyl-T) base pair anions. J. Am. Chem. Soc. 127, 6443-6450 (2005).

13. Mazurkiewicz, K. et al. Intermolecular proton transfer induced by excess electron attachment to adenine(formic acid)n $(n=2,3)$ hydrogen-bonded complexes. Chem. Phys. 342, 215-222 (2007)

14. Ko, Y. J. et al. Barrier-free proton transfer induced by electron attachment to the complexes between 1-methylcytosine and formic acid. Mol. Phys. 108, 2621-2631 (2010).

15. Harańczyk, M. et al. Excess electron attachment induces barrier-free proton transfer in anionic complexes of thymine and uracil with formic acid. J. Phys. Chem. B 108, 6919-6921 (2004).

16. Harańczyk, M. et al. Intermolecular proton transfer in anionic complexes of uracil with alcohols. J. Phys. Chem. B 109, 13383-13391 (2005).

17. Harańczyk, M. et al. Excess electron attachment induces barrier-free proton transfer in binary complexes of uracil with $\mathrm{H}_{2} \mathrm{Se}$ and $\mathrm{H}_{2} \mathrm{~S}$ but not with $\mathrm{H}_{2} \mathrm{O}$. J. Phys. Chem. B 107, 7889-7895 (2003).

18. Harańczyk, M. et al. Effect of hydrogen bonding on barrier-free proton transfer in anionic complexes of uracil with weak acids: $(\mathrm{U} . . . \mathrm{HCN})^{-}$versus $\left(\mathrm{U} . . . \mathrm{H}_{2} \mathrm{~S}\right)^{-}$. Isr. J. Chem. 44, 157-170 (2004).

19. Rak, J. et al. Stable valence anions of nucleic acid bases and DNA strand breaks induced by low energy electrons. In Radiation Induced Molecular Phenomena in Nucleic Acids: A Comprehensive Theoretical and Experimental Analysis (eds Shukla, M. K. \& Leszczynski, J.) 619-667 (Springer Netherlands, Dordrecht, Netherlands, 2008)

20. Keolopile, Z. G. et al. Importance of time scale and local environment in electron-driven proton transfer. The anion of acetoacetic acid. J. Am. Chem. Soc. 137, 14329-14340 (2015).

21. Zhao, J., Ji, S., Chen, Y., Guo, H. \& Yang, P. Excited state intramolecular proton transfer (ESIPT): from principal photophysics to the development of new chromophores and applications in fluorescent molecular probes and luminescent materials. Phys. Chem. Chem. Phys. 14, 8803-8817 (2012).

22. Collins, K. D., Gensch, T. \& Glorius, F. Contemporary screening approaches to reaction discovery and development. Nat. Chem. 6, 859-871 (2014).

23. Lee, J., Kim, C. H. \& Joo, T. Active role of proton in excited state intramolecular proton transfer reaction. J. Phys. Chem. A 117, 1400-1405 (2013).

24. Martinez, M. L., Cooper, W. C. \& Chou, P.-T. A novel excited-state intramolecular proton transfer molecule, 10-hydroxybenzo[h]quinoline. Chem. Phys. Lett. 193, 151-154 (1992).

25. Chou, P.-T. \& Wei, C.-Y. Photophysics of 10-hydroxybenzo[h]quinoline in aqueous solution. J. Phys. Chem. 100, 17059-17066 (1996).
26. Chou, P.-T. et al. Excited-state intramolecular proton transfer in 10-hydroxybenzo[h]quinoline. J. Phys. Chem. A 105, 1731-1740 (2001).

27. Takeuchi, S. \& Tahara, T. Coherent nuclear wavepacket motions in ultrafast excited-state intramolecular proton transfer: sub-30-fs resolved pump-probe absorption spectroscopy of 10-hydroxybenzo[h] quinoline in solution. J. Phys. Chem. A 109, 10199-10207 (2005).

28. Schriever, C. et al. The interplay of skeletal deformations and ultrafast excitedstate intramolecular proton transfer: experimental and theoretical investigation of 10-hydroxybenzo[h]quinoline. Chem. Phys. 347, 446-461 (2008).

29. Kim, C. H. \& Joo, T. Coherent excited state intramolecular proton transfer probed by time-resolved fluorescence. Phys. Chem. Chem. Phys. 11, 10266-10269 (2009).

30. Higashi, M. \& Saito, S. Direct simulation of excited-state intramolecular proton transfer and vibrational coherence of 10-hydroxybenzo[h] quinoline in solution. J. Phys. Chem. Lett. 2, 2366-2371 (2011).

31. Chansen, W. et al. Theoretical study on influence of geometry controlling over the excited state intramolecular proton transfer of 10-hydroxybenzo[h] quinoline and its derivatives. Comput. Theor. Chem. 1113, 42-51 (2017).

32. Lan, S.-C. \& Liu, Y.-H. TDDFT study on the excited-state proton transfer of 8hydroxyquinoline: key role of the excited-state hydrogen-bond strengthening. Spectrochim. Acta A 139, 49-53 (2015).

33. Wu, J., Liu, W., Ge, J., Zhang, H. \& Wang, P. New sensing mechanisms for design of fluorescent chemosensors emerging in recent years. Chem. Soc. Rev. 40, 3483-3495 (2011).

34. Kwon, J. E. \& Park, S. Y. Advanced organic optoelectronic materials: harnessing excited-state intramolecular proton transfer (ESIPT) process. $A d v$. Mater. 23, 3615-3642 (2011).

35. Park, S. et al. A white-light-emitting molecule: frustrated energy transfer between constituent emitting centers. J. Am. Chem. Soc. 131, 14043-14049 (2009).

36. Tang, K.-C. et al. Fine tuning the energetics of excited-state intramolecular proton transfer (ESIPT): white light generation in a single ESIPT system. J. Am. Chem. Soc. 133, 17738-17745 (2011).

37. Hsieh, C.-C., Jiang, C.-M. \& Chou, P.-T. Recent experimental advances on excited-state intramolecular proton coupled electron transfer reaction. Acc. Chem. Res. 43, 1364-1374 (2010).

38. Luxami, V. \& Kumar, S. Molecular half-subtractor based on 3,3[prime or minute]-bis(1H-benzimidazolyl-2-yl)[1,1[prime or minute]]binaphthalenyl2,2[prime or minute]-diol. New J. Chem. 32, 2074-2079 (2008).

39. Grubisic, A. et al. $\mathrm{Al}_{13} \mathrm{H}^{-}$: hydrogen atom site selectivity and the shell model. J. Chem. Phys. 131, 121103 (2009).

40. Paterson, M. J., Robb, M. A., Blancafort, L. \& DeBellis, A. D. Mechanism of an exceptional class of photostabilizers: a seam of conical intersection parallel to excited state intramolecular proton transfer (ESIPT) in o-Hydroxyphenyl(1,3,5)-triazine. J. Phys. Chem. A 109, 7527-7537 (2005).

41. Patil, V. S., Padalkar, V. S., Tathe, A. B., Gupta, V. D. \& Sekar, N. Synthesis, photo-physical and DFT studies of ESIPT inspired novel 2- $\left(2^{\prime}, 4^{\prime}-\right.$ Dihydroxyphenyl) benzimidazole, benzoxazole and benzothiazole. J. Fluoresc. 23, 1019-1029 (2013).

42. Ameer-Beg, S. et al. Ultrafast measurements of excited state intramolecular proton transfer (ESIPT) in room temperature solutions of 3-hydroxyflavone and derivatives. J. Phys. Chem. A 105, 3709-3718 (2001).

43. Sobolewski, A. L. \& Domcke, W. Computational studies of the photophysics of hydrogen-bonded molecular systems. J. Phys. Chem. A 111, 11725-11735 (2007).

44. Zhang, X. et al. On the existence of designer magnetic superatoms. J. Am. Chem. Soc. 135, 4856-4861 (2013).

45. Ho, J., Ervin, K. M. \& Lineberger, W. C. Photoelectron spectroscopy of metal cluster anions: $\mathrm{Cu}_{\mathrm{n}}{ }^{-}, \mathrm{Ag}_{\mathrm{n}}{ }^{-}$, and $\mathrm{Au}_{\mathrm{n}}{ }^{-}$. J. Chem. Phys. 93, 6987-7002 (1990).

\section{Acknowledgements}

X.Z. acknowledges the College of Chemistry at Nankai University for the start funding. This material is based upon work supported by the (U.S.) National Science Foundation under Grant No. CHE-1664182.

\section{Author contributions}

X.Z. designed the experiments. X.Z., M.M., E.C. and S.M. performed the experiments and interpreted the data. W.W. and C.M. performed the theoretical calculations. X.Z. and W.W. wrote the manuscript. K.H.B. revised the manuscript. X.Z. and K.H.B. supervised the whole work 


\section{Additional information}

Supplementary Information accompanies this paper at https://doi.org/10.1038/s41467019-09154-5.

Competing interests: The authors declare no competing interests.

Reprints and permission information is available online at http://npg.nature.com/ reprintsandpermissions/

Journal peer review information: Nature Communications thanks the anonymous reviewers for their contribution to the peer review of this work.

Publisher's note: Springer Nature remains neutral with regard to jurisdictional claims in published maps and institutional affiliations. (c) (i) Open Access This article is licensed under a Creative Commons Attribution 4.0 International License, which permits use, sharing, adaptation, distribution and reproduction in any medium or format, as long as you give appropriate credit to the original author(s) and the source, provide a link to the Creative Commons license, and indicate if changes were made. The images or other third party material in this article are included in the article's Creative Commons license, unless indicated otherwise in a credit line to the material. If material is not included in the article's Creative Commons license and your intended use is not permitted by statutory regulation or exceeds the permitted use, you will need to obtain permission directly from the copyright holder. To view a copy of this license, visit http://creativecommons.org/ licenses/by/4.0/.

(C) The Author(s) 2019 